\title{
Mass correlation between light and heavy reaction products in multinucleon transfer ${ }^{197} \mathrm{Au}+{ }^{130} \mathrm{Te}$ collisions
}

\author{
F. Galtarossa, ${ }^{1,2}$ L. Corradi, ${ }^{1}$ S. Szilner,${ }^{3}$ E. Fioretto, ${ }^{1}$ G. Pollarolo, ${ }^{4}$ T. Mijatović,,${ }^{3}$ D. Montanari, ${ }^{5}$ D. Ackermann, ${ }^{6}$ \\ D. Bourgin, ${ }^{7}$ S. Courtin, ${ }^{7}$ G. Fruet, ${ }^{7}$ A. Goasduff, ${ }^{1}$ J. Grebosz, ${ }^{8}$ F. Haas, ${ }^{7}$ D. Jelavić Malenica, ${ }^{3}$ S. C. Jeong, ${ }^{9}$ H. M. Jia,${ }^{10}$ \\ P. R. John, ${ }^{5}$ D. Mengoni, ${ }^{5}$ M. Milin, ${ }^{11}$ G. Montagnoli, ${ }^{5}$ F. Scarlassara,${ }^{5}$ N. Skukan, ${ }^{3}$ N. Soić, ${ }^{3}$ A. M. Stefanini, ${ }^{1}$ E. Strano, ${ }^{5}$ \\ V. Tokić, ${ }^{3}$ C. A. Ur, ${ }^{12}$ J. J. Valiente-Dobón, ${ }^{1}$ and Y. X. Watanabe ${ }^{9}$ \\ ${ }^{1}$ Istituto Nazionale di Fisica Nucleare, Laboratori Nazionali di Legnaro, Legnaro, Italy \\ ${ }^{2}$ Dipartimento di Fisica e Scienze della Terra, Università di Ferrara, Ferrara, Italy \\ ${ }^{3}$ Ruđer Bošković Institute Zagreb, Croatia \\ ${ }^{4}$ Dipartimento di Fisica, Università di Torino, and Istituto Nazionale di Fisica Nucleare, Torino, Italy \\ ${ }^{5}$ Dipartimento di Fisica, Università di Padova, and Istituto Nazionale di Fisica Nucleare, Padova, Italy \\ ${ }^{6}$ GANIL, CEA/DSM-CNRS/IN2P3, Boulevard Henri Becquerel, Caen, France \\ ${ }^{7}$ Institut Pluridisciplinaire Hubert Curien, CNRS-IN2P3, Université de Strasbourg, Strasbourg, France \\ ${ }^{8}$ The Henryk Niewodniczanski Institute of Nuclear Physics, Krakow, Poland \\ ${ }^{9}$ Institute of Particle and Nuclear Studies, High Energy Accelerator Research Organization (KEK), Tsukuba, Ibaraki, Japan \\ ${ }^{10}$ China Institute of Atomic Energy, Bejing, China \\ ${ }^{11}$ Department of Physics, Faculty of Science, University of Zagreb, Zagreb, Croatia \\ ${ }^{12}$ Horia Hulubei National Institute of Physics and Nuclear Engineering, Bucharest, Romania
}

(Received 8 November 2017; revised manuscript received 10 April 2018; published 17 May 2018)

\begin{abstract}
We studied multinucleon transfer reactions in the ${ }^{197} \mathrm{Au}+{ }^{130} \mathrm{Te}$ system at $E_{\mathrm{lab}}=1.07 \mathrm{GeV}$ by employing the PRISMA magnetic spectrometer coupled to a coincident detector. For each light fragment we constructed, in coincidence, the distribution in mass of the heavy partner of the reaction. With a Monte Carlo method, starting from the binary character of the reaction, we simulated the de-excitation process of the produced heavy fragments to be able to understand their final mass distribution. The total cross sections for pure neutron transfer channels have also been extracted and compared with calculations performed with the GRAZING code.
\end{abstract}

DOI: 10.1103/PhysRevC.97.054606

\section{INTRODUCTION}

The production of neutron-rich heavy nuclei in the region of lead, i.e., near the $N=126$ shell closure, received in recent years a boost of interest since the properties of these nuclei are fundamental for the understanding of the actual path, in the $(N, Z)$ plane, that is chosen by the $r$-process to synthesize the heavy elements. Unfortunately the investigation of the nuclei in this region is hampered by the difficulties in their production and in their direct identification with present techniques.

For their production a promising mechanism is provided by the exploitation of nucleon transfer reactions between heavy ions, for which nuclear reaction models $[1,2]$ predict large primary multinucleon transfer cross sections that are comparable, or even larger, than those of other reactions like fragmentation at intermediate energies [3] or low-energy fusion with radioactive beams [4].

At energies close to the Coulomb barrier, multinucleon transfer reactions are mainly governed by optimum $Q$-value considerations and nuclear form factors $[5,6]$. Such ingredients allow one to understand how nucleons are exchanged between projectile and target and how energy and angular momentum are transferred from the relative motion to the intrinsic excitation. On this basis one understands why, with stable nuclei, the dominant channels are the neutron pick-up and the proton stripping [6-8], where the light partner of the reaction is the projectile, so that the reaction tends to populate heavy partner products that are neutron poor. With neutron-rich projectiles it has been predicted in Ref. [1] a change in the population pattern that leads to primary neutron-rich heavy partners (i.e., the transition probability is larger for the neutron-stripping channel). It may be interesting to stress that Refs. [1,2] are envisaging very different mechanisms for the production of heavy neutron-rich nuclei. While in the former these nuclei are produced via a direct reaction in the latter the production is seen in the tails of the mass and charge distributions of the decay of a dinuclear complex. The emitting angles and energies of the fragments are very different in the two cases, which implies that while in the first case we can use a two-body kinematic to reconstruct the reaction this cannot be done in the second case.

The path leading to primary neutron-rich heavy partners, in multinucleon transfer reactions, has been investigated in recent high-resolution experiments with neutron-rich stable projectiles, in ${ }^{64} \mathrm{Ni}+{ }^{238} \mathrm{U}[9],{ }^{40} \mathrm{Ar}+{ }^{208} \mathrm{~Pb}[10],{ }^{136} \mathrm{Xe}+$ ${ }^{198} \mathrm{Pt}$ [11], and ${ }^{136} \mathrm{Xe}+{ }^{238} \mathrm{U}$ [12]. In these reactions the light partner has been identified directly via high-resolution magnetic spectrometers while, in some of the mentioned cases, information on the heavy partner was obtained indirectly by detecting the coincident $\gamma$ rays produced by the fragments. In these experiments information on secondary processes, i.e., 
neutron evaporation, could also be extracted as illustrated for example in Refs. [13-15]. In Ref. [11] in particular it has been shown that the most neutron-rich nuclei in the $\mathrm{Hg}$-Os region are produced mainly through collisions involving small kinetic energy losses. Investigations carried out with radiochemical methods in heavy actinides [16] also indicate that neutron-rich nuclei are produced in the low-energy tails of the dissipated energy distributions. In all these experiments it has been emphasized that secondary processes may significantly influence the final yield distributions, even though rather meager evidence of the survival probability of the heavy partner has been provided. These issues have been also investigated in high-efficiency but low-resolution measurements employing particle coincidence techniques $[17,18]$.

In order to understand and quantify the production process also for the heavy partner of the reaction, we performed an experiment with a simultaneous detection of light and heavy transfer products in the ${ }^{197} \mathrm{Au}+{ }^{130} \mathrm{Te}$ system. We chose the neutron-rich ${ }^{130} \mathrm{Te}$ to populate neutron transfer channels leading primarily to neutron-rich $\mathrm{Au}$ isotopes. We exploited the performance of the PRISMA spectrometer [13] to identify isotopes in the tellurium region, while the coincident Au-like partners were detected with a dedicated set-up [19] (here referred to as NOSE) specifically built and coupled to PRISMA. This allowed, via a high-resolution mass-mass correlation, to study the final mass distribution of the heavy partner and the effect of secondary processes. We focused on pure neutron transfer channels being the cross sections for proton transfers almost an order of magnitude lower at the chosen low bombarding energy. Favorable experimental conditions were achieved by employing inverse kinematics in such a way that both the light recoil and the heavy partner could have enough energy for their detection.

\section{THE SET-UP AND THE EXPERIMENT}

Figure 1 schematically shows the layout of the experimental set-up. The PRISMA detector system, which in the present experiment has been employed for the detection of the tellurium ions, provides all the necessary information for the complete ion identification, which is performed via an event-by-event reconstruction of the ion trajectory inside the magnetic elements. A start signal for time-of-flight measurements and two-dimensional position information are given by a position-sensitive microchannel plate detector (MCP). After passing through a quadrupole and a dipole magnetic element, ions enter a focal plane detector made of a parallelplate detector of multiwire type (MWPPAC), providing timing and position signals, followed by an array of transverse-field multiparametric ionization chambers, where the nuclear charge $Z$ is identified via energy loss $\left(\Delta E_{\mathrm{IC}}\right)$ and total energy $\left(E_{\mathrm{IC}}\right)$ measurements.

The coincident detector (NOSE), providing information on the heavy partner of the reaction, consists of a multiwire parallel-plate avalanche counter (referred to as PPAC) followed by an axial-field ionization chamber (Bragg chamber). The former provides timing and two-dimensional position information, the latter identifies the fragments in terms of nuclear charge (via Bragg peak), range and total energy $\left(E_{\mathrm{BC}}\right)$. The

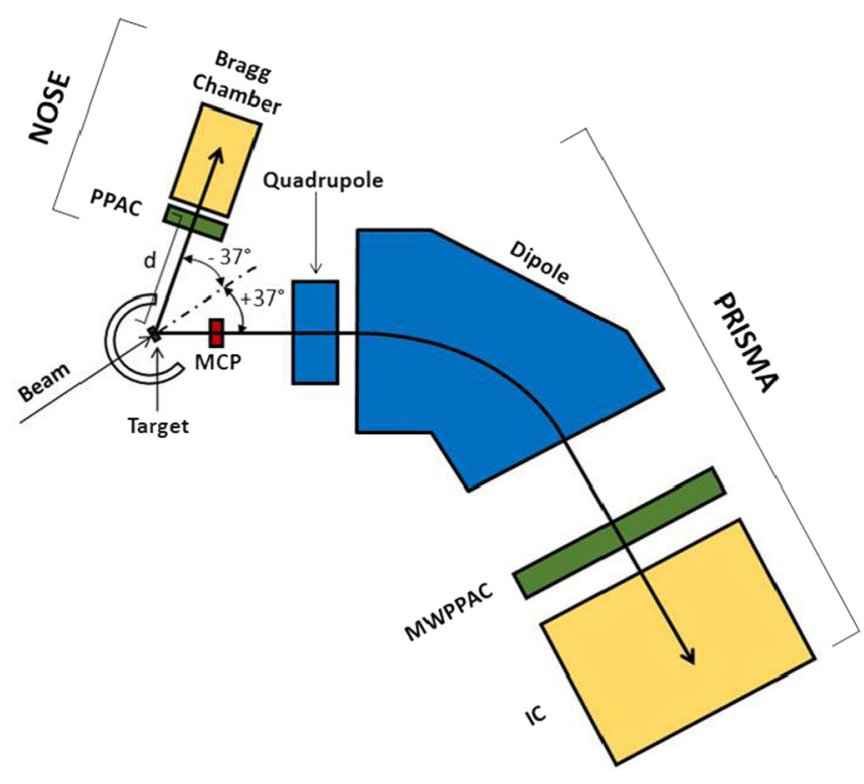

FIG. 1. Layout of the set-up used in the study of the ${ }^{197} \mathrm{Au}+{ }^{130} \mathrm{Te}$ reaction, with the PRISMA spectrometer set in coincidence with the NOSE detector. Bragg chamber: axial-field ionization chamber; PPAC: multiwire parallel-plate avalanche counter of NOSE; MCP: microchannel plate detector; MWPPAC: parallel-plate detector of multiwire type; IC: ionization chamber of PRISMA.

detector system has a geometrical solid angle about one third of PRISMA. The resolutions of the NOSE detectors are similar to those of PRISMA, in particular $\sim 1 \mathrm{~mm}$ for the positions and $\sim 350 \mathrm{ps}$ for the timing. The time difference between MCP and PPAC signals $(\triangle \mathrm{ToF})$ was used to construct the time-of-flight over the distance $d \sim 90 \mathrm{~cm}$ between the target and the PPAC of NOSE, referred also as crossing time. This was done by taking into account the flight time of the light partner identified in PRISMA over the distance of $25 \mathrm{~cm}$ between the target and the MCP. The cathodes of the MWPPAC acted as master trigger for the data acquisition system.

For the experiment we used a 1.5-pnA ${ }^{197}$ Au beam delivered by the PIAVE positive-ion injector followed by the ALPI post accelerator of LNL, impinging onto a $200 \mu \mathrm{g} / \mathrm{cm}^{2}$ (2-mm strip) ${ }^{130} \mathrm{Te}$ target with a purity of $99.6 \%$. To reduce the sputtering effects on the target surface, the Te material was sandwiched between $20 \mu \mathrm{g} / \mathrm{cm}^{2}$ carbon layers. The bombarding energy was $E_{\text {lab }}=1.07 \mathrm{GeV}$. PRISMA and NOSE were symmetrically placed at $+37^{\circ}$ and $-37^{\circ}$ for Te-like and Au-like ions, respectively, this angle corresponds to the maximum of the cross section for the one-neutron transfer channel as predicted by the GRAZING code [20-22]. In this configuration Te-like recoils entering PRISMA (whose geometrical in-plane angular acceptance is $\pm 6^{\circ}$ ) had energies of $\sim 5.0 \mathrm{MeV} / A$, while $\mathrm{Au}$-like ions in NOSE had energies of $\sim 2.2 \mathrm{MeV} / A$, these last corresponding to a velocity of $\sim 2.07 \mathrm{~cm} / \mathrm{ns}$ and a crossing time of $\sim 43 \mathrm{~ns}$. The energy loss variation of Au-like ions in the target leads to a contribution to the mass resolution of the heavy partner of about $1 \%$, a value comparable to that due to the intrinsic time resolution of the detectors. Both detection systems were connected to a sliding-seal scattering chamber 


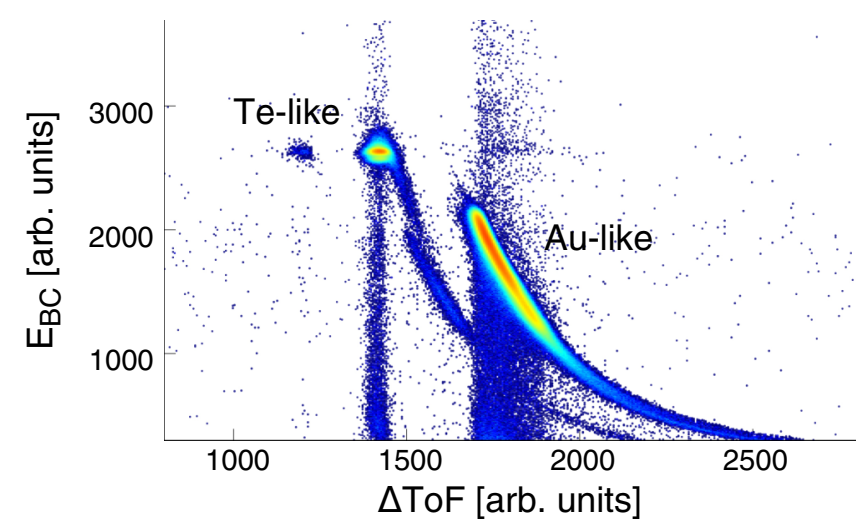

FIG. 2. Two-dimensional matrix of total energy measured with the Bragg chamber $\left(E_{\mathrm{BC}}\right)$ vs the time-of-flight between the MCP of PRISMA and the PPAC of NOSE $(\triangle T$ ToF). The clearly separated Telike and Au-like events are labeled. The repetition of the Au-like and Te-like structures on their respective left sides are due to electronic effects in the time-of-flight while the vertical bands correspond to uncorrelated events. Overall, these spurious structures are only a few percent of the total events.

( $~ 0.5$ m diameter) inside which two monitors of silicon surface barrier type were placed at $\theta_{\mathrm{lab}}=61^{\circ}$ and $\theta_{\mathrm{lab}}=71^{\circ}$. The monitors were used to detect Rutherford-scattered Te ions for relative normalization between different runs and to control the beam conditions during the measurements.

\section{RESULTS}

Indicating the collision as $A+a \rightarrow B+b$ where $(A+a)$ is the entrance channel ( $A$ being the heavy projectile) and $(B+$ $b$ ) the exit channel ( $B$ being the projectile-like fragment) we can write the mass $M_{B}$ of the heavy projectile-like fragment in the form

$$
M_{B}=\frac{p_{A}}{d} \frac{\sin \theta_{b}}{\sin \left(\theta_{B}+\theta_{b}\right)} \tau_{B},
$$

where $p_{A}$ is the linear momentum of the projectile in the laboratory system. The parameter $\tau_{B}$ indicates the crossing time over the distance $d$ in the NOSE detector. The scattering angles $\theta_{b}$ and $\theta_{B}$ correspond to the light and heavy fragments measured in PRISMA and in NOSE, respectively. We note that the mass resolution strongly depends on the precision with which we measure the crossing time in the NOSE apparatus and that the correlation with the mass of the light fragment enters through the scattering angle. We also point out that, since both $p_{A}$ and $\tau_{B}$ depend on the interaction point of the ions into the target, its thickness may have a considerable effect on the mass resolution. Of course the above correlation formula has been obtained by assuming a binary character of the reaction.

The fact that with PRISMA and NOSE detectors, placed in the configuration discussed above, we are able to select binary events, can be appreciated by the plots shown in Figs. 2 and 3 , which display the event distributions in the matrix $E_{\mathrm{BC}}$ vs $\Delta \mathrm{ToF}$ and $\Delta E_{\mathrm{IC}}-E_{\mathrm{IC}}$ of PRISMA, respectively. In Fig. 2 one can clearly observe the well-separated events belonging to the Te-like and Au-like ions. In Fig. 3 (bottom panel) one sees the

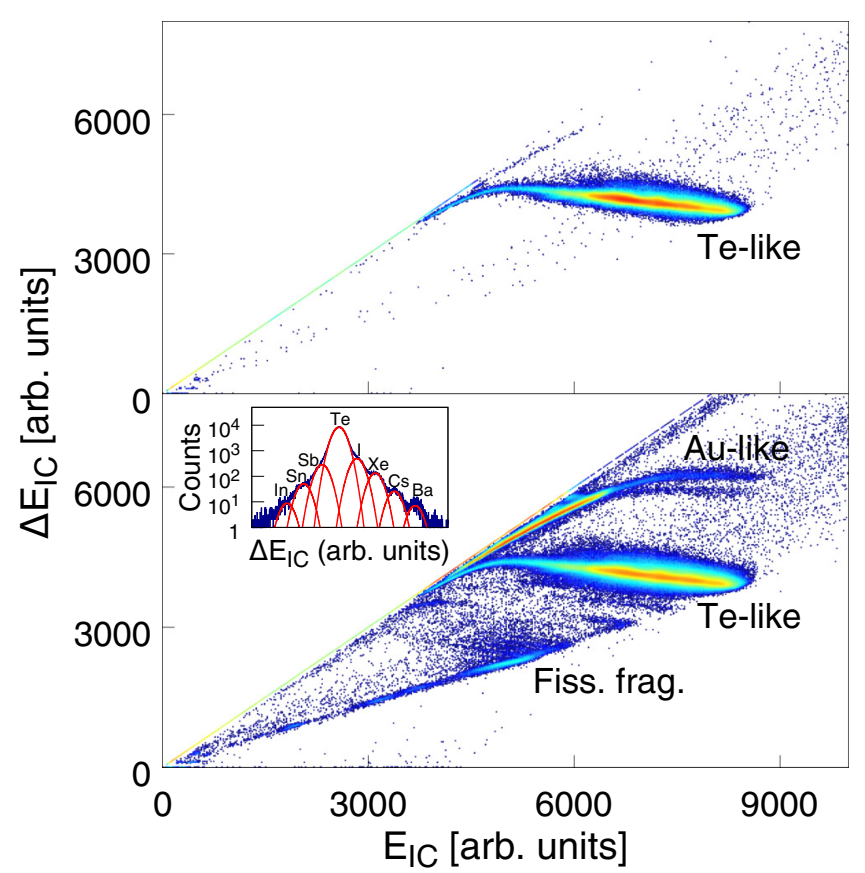

FIG. 3. (Bottom) Matrix of $\Delta E_{\mathrm{IC}}$ vs energy $E_{\mathrm{IC}}$ of the PRISMA ionization chamber for the ${ }^{197} \mathrm{Au}+{ }^{130} \mathrm{Te}$ reaction at $E_{\text {lab }}=1.07 \mathrm{GeV}$ and $\theta_{\text {lab }}=37^{\circ}$. The most intense band corresponds to Te-like ions, while $\mathrm{Au}$-like ions are located in the region where $\Delta E_{\mathrm{IC}}$ and $E_{\mathrm{IC}}$ merge. Ions corresponding to fission events, which are not stopped in the IC, are also labeled. The $Z$ distributions of the reaction products in the Te region are labeled in the inset. (Top) The same events as below but in coincidence with the Au-like events in the NOSE detector. Notice that the only remaining events are the Te-like ones indicating clearly that they are binary in nature.

most intense band corresponding to the Te-like ions, also well separated from the other events, in particular Au-like ions and ions with an average nuclear charge $Z \sim 39$, that are coming from fission. In a binary reaction the Au-like events in NOSE must be uniquely associated to the Te-like events detected in PRISMA, this is seen in the top panel of Fig. 3 where, in coincidence with the Au-like events in NOSE, all events in the ionization chamber of the spectrometer are shown.

As a further argument to demonstrate that we are analyzing events belonging to binary reactions we show in Fig. 4 the correlation between the two scattering angles of the fragments in comparison with the kinematic calculations for elastic+inelastic scattering. The distributions of the experimental data are clearly consistent with the kinematic calculations. The events we are selecting are thus binary and derive from quasielastic reactions, i.e., reactions coming from a small region of partial waves close to the grazing angular momentum. The fission-like events seen in Fig. 3, with a measured yield of less than 5\%, that are not in coincidence with the Au-like events in NOSE, are coming from reactions with partial waves smaller than the grazing one and thus with large dissipated energy. In the inset of Fig. 3 (bottom) is shown the yield distribution of the events as a function of the nuclear charge $Z$ (mass integrated) that has been obtained after a linearization of the Te-like events. Because of $Q$-value reasons the proton transfer channels, at 


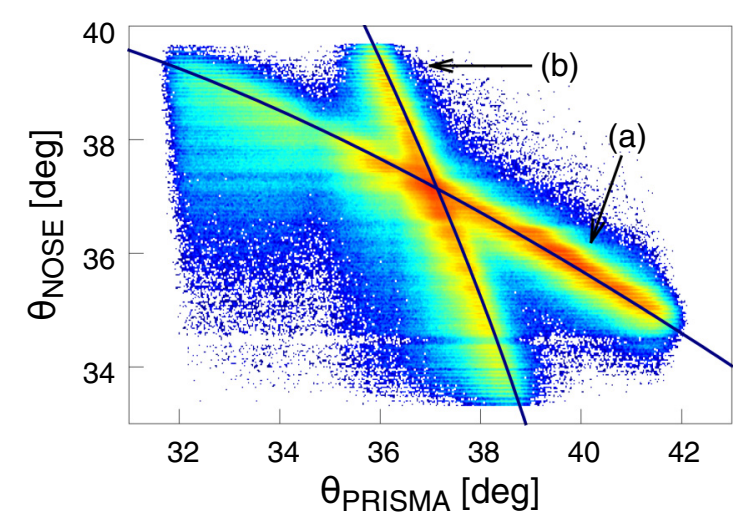

FIG. 4. Correlation matrix of the in-plane scattering angles measured with the PPAC of NOSE $\left(\theta_{\text {NOSE }}\right)$ vs MCP of PRISMA $\left(\theta_{\text {PRISMA }}\right)$. The band (a) corresponds to the Te-like ions detected in PRISMA and the Au-like ions detected in the second arm, the band (b) to the reverse combination. The curves indicate the calculated elastic+inelastic scattering. The difference in intensity of the two bands reflects the setting of PRISMA that has been optimized for the Te-like ions.

this low bombarding energy, lie in an energy range close to the overwhelming elastic component so that the nuclear charge selection turns out to be quite difficult. However the stripping and pick-up of protons are visible and they have a cross section that is quite small. This is why the proton transfer channels are left out from any further considerations.

For the mass identification of the reaction products one needs to reconstruct their trajectory in the magnetic elements of PRISMA. The reconstruction of the trajectory is obtained from the measurement of the position in the entrance and focal-plane detectors and from the corresponding time of flight. The procedure has to take into account the distribution of the atomic charge states $q$ of the ions in order to convert the mass over atomic charge state $(A / q)$ distributions to the final mass distributions. Partial overlaps in $A / q$, especially relevant for weakly populated transfer channels in the presence of an overwhelming elastic channel, could be better recognized

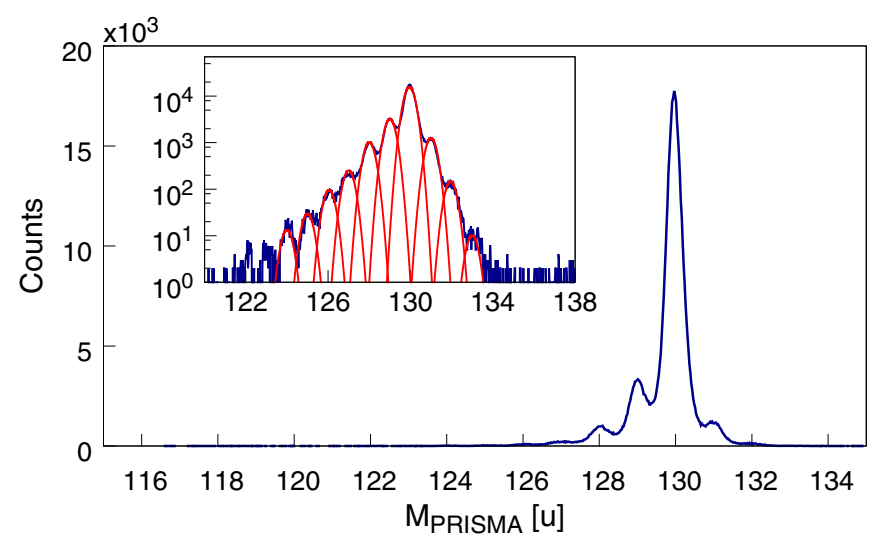

FIG. 5. Mass distribution for the Te isotopes obtained after ion trajectory reconstruction in PRISMA. The inset shows the distribution in logarithmic scale with the multi-Gaussian fit used to evaluate the yields of the neutron transfer channels. and eliminated thanks to the information derived from the kinematic coincidence. From the linearized $A / q$ distributions we extracted the mass spectrum summing over all atomic charge states. The outcome of this procedure is the final mass distribution for the Te isotopes depicted in Fig. 5 where the resolution turned out to be $\Delta A / A \sim 1 / 240$, allowing to separate the different isotopes.

From Fig. 5 one sees how the dominant flux is in the neutron pick-up side, whose yield could be measured down to the $(6 n)$


FIG. 6. Total kinetic energy loss (TKEL) distributions for the indicated neutron pick-up transfer channels measured in PRISMA (black) and by imposing a kinematic coincidence with Au-like events in NOSE (blue). The vertical lines correspond to the ground-state-toground-state $Q$ values. 


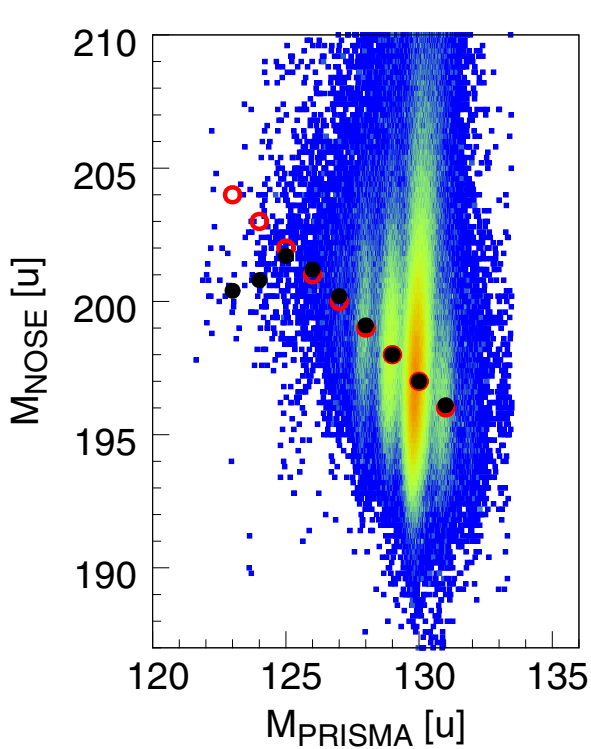

FIG. 7. Mass-mass correlation matrix of Te isotopes detected in PRISMA and the heavy partner detected in coincidence with NOSE. The red circles indicate the centroids of the correlated masses of the primary neutron transfer channels, the black dots indicate the experimental centroids as derived from the fits of their projections (see also Fig. 8).

channel. The terms stripping and pick-up are referred in the usual way to the projectile, i.e., to the Au.

To understand the amount of energy that is dissipated in the process and thus the amount of excitation energy that is present in the fragments we study the total kinetic energy loss (TKEL) distributions for the different channels. These TKEL distributions were constructed by using solely the data extracted with the PRISMA spectrometer and imposing the linear momentum conservation proper of a binary reaction. In Fig. 6 the TKEL distributions obtained for some representative neutron pick-up transfer channels are shown with and without requiring that a Te in PRISMA is in coincidence with a Au-like event in the NOSE. The TKEL distributions display a well defined peak, which for few-neutron transfer channels is close to the optimum $Q$ value $\left(Q_{\mathrm{opt}} \sim 0 \mathrm{MeV}\right)$, as expected in a regime where quasi-elastic processes dominate. The high-energy tails indicate that more complicated processes like deep inelastic (DIC) may contribute. The shapes of the distributions with and without the requirement of coincidence are almost the same and their intensity ratios, reflecting the relative geometrical solid angles of the two detectors, are nearly constant for all the shown transfer channels.

Now, via Eq. (1) we can construct the matrix that correlates the mass of the Te isotopes measured with PRISMA and the mass of the associated Au-like nuclei detected in coincidence with NOSE. This is shown in Fig. 7. Here we see that the identification in mass of the light fragment with high resolution allows to separate the mass distribution of the heavy partner in well-defined bands. The black dots in Fig. 7 indicate the centroids of the projections of each band, showing how they slightly bend toward lower masses in comparison to those expected for the corresponding primary neutron transfer

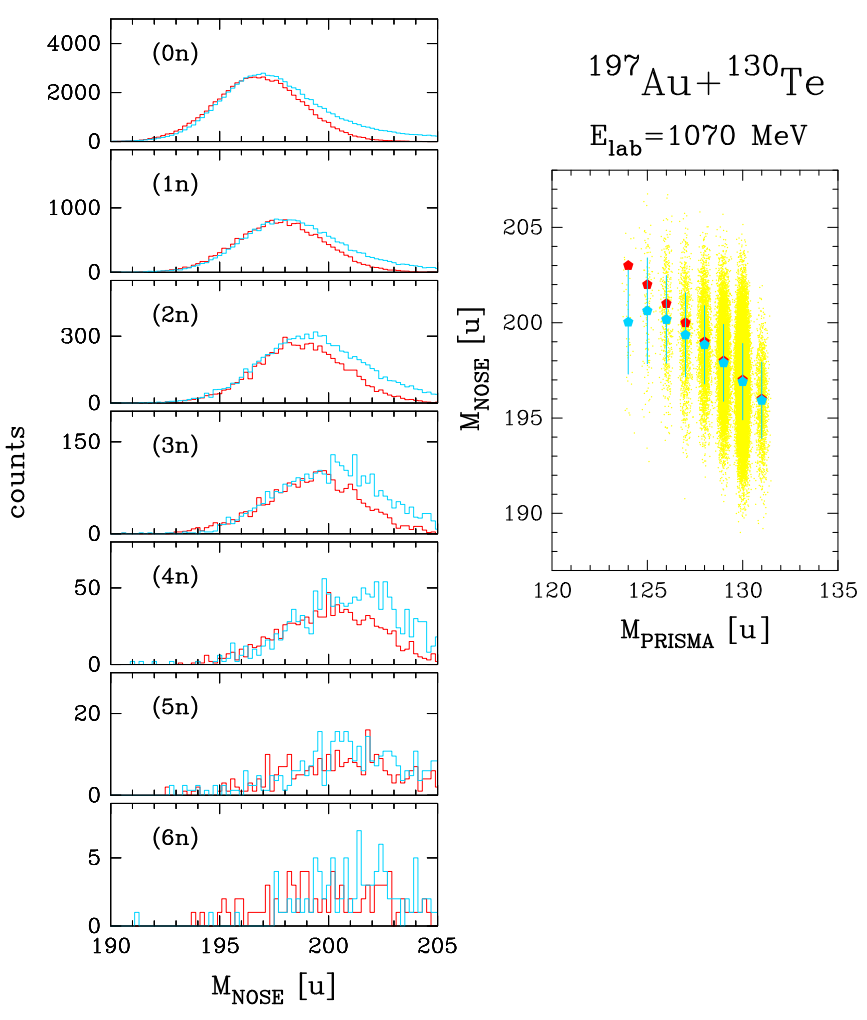

FIG. 8. Right: simulated mass-mass correlation matrix. The points are the centroids of the primary (red) and actual (blue/light gray) $\mathrm{Au}$ isotope distributions. The blue/light gray bars represent the standard deviations. Left: comparison between the simulated (red) and experimental (blue/light gray) mass distributions obtained from the projection of the corresponding mass-mass matrix. The label in each frame indicates the number of neutrons stripped from ${ }^{130} \mathrm{Te}$.

channels (red circles). This indicates that the primary fragments acquire significant excitation energy so that evaporation becomes relevant in defining the final yields. The shift of the centroids towards lower masses may be better appreciated in the projections of the matrix, as seen in Fig. 8.

The neutron evaporation, besides shifting the centroids, will enlarge the width of the distributions, that are also influenced by the experimental resolution. To disentangle the contribution of these two components we made a Monte Carlo simulation of the behavior of the binary process. In the simulation we started from a given event as recorded by PRISMA. So we know the scattering angle, energy and mass of the target-like fragments. By using a binary kinematic we associate to it a projectile-like fragment with a given mass (from the mass conservation), a given momentum and a given scattering angle. To the projectile-like fragment we assign also an excitation energy (extracted from the TKEL spectra assuming an equal sharing of the energy among the two fragments) and then with a very simple evaporation model we followed its decaying process up to the NOSE detector. To construct the matrix of the mass-mass correlation we distributed the initial events in accordance to the cross sections measured by PRISMA for the Te isotopes. To obtain the shown good comparison we had to include, in the simulation, a $1 \%$ error on the crossing time $\tau_{B}$, which corresponds to the mass resolution obtained for the 

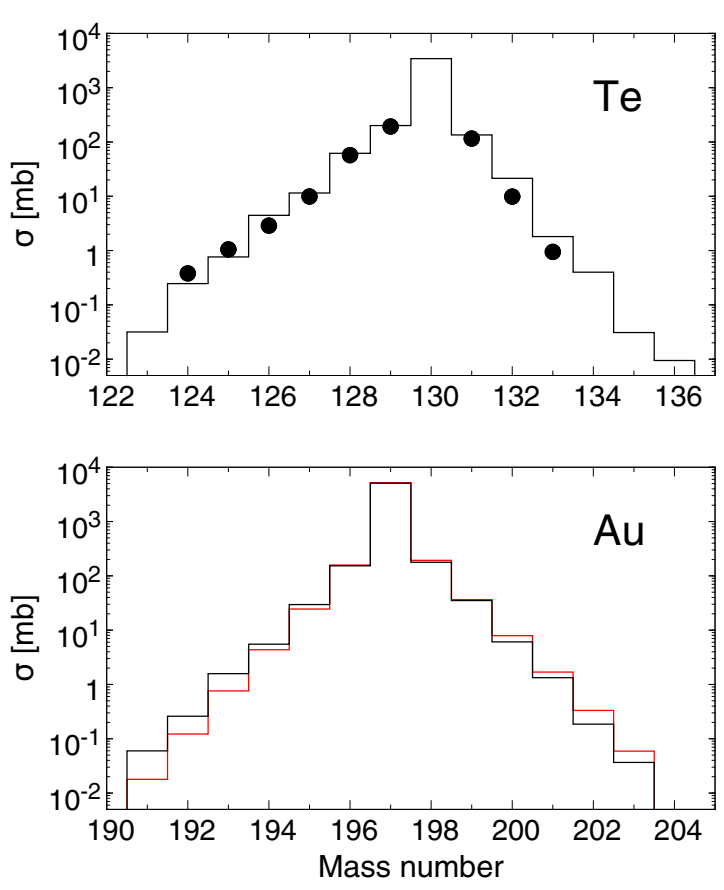

FIG. 9. (Top) Total experimental cross sections of Te isotopes (points) and GRAZING calculations (histogram). Errors are only statistical and are within symbols. (Bottom) GRAZING predictions for the $\mathrm{Au}$ isotopes with (black histogram) and without (red histogram) neutron evaporation.

heavy partner $\Delta A / A \sim 1 / 40$. We stress that it is this error that dominates the width of the distributions. The results of the simulation are reported in Fig. 8 (right frame) while on the lefthand side we show, for the different channels, the projection on the heavy-mass axis in comparison with the histograms of the experimental data. The simulations indicate also that the shift of the centroids towards lower masses is due to evaporation. From these shifts we could extract information on the average number of evaporated neutrons for each channel associated with the Te isotopes.

As last part of this work we feel important to compare the extracted cross sections with the ones calculated with the GRAZING code [20-22] that implements a model of the collision that is predominantly binary. This model calculates the evolution of the reaction by taking into account, besides the relative motion variables, the intrinsic degrees of freedom of projectile and target. These are the surface degrees of freedom and the one-nucleon transfer channels. The relative motion of the system is calculated in a nuclear plus Coulomb field. The exchange of many nucleons proceeds via a multistep mechanism of single nucleons. This model has been successfully applied in the description of multinucleon transfer reactions [6] as well as of fusion reactions and barrier distributions [23].

Figure 9 (top panel) displays the experimental cross sections (points) together with the calculations done with the GRAZING code (histogram). To normalize the experimental data to the calculation we exploited the fact that the angular distributions for all neutron transfer channels have very similar shapes and present their maximum all at the same angle (PRISMA has a quite large acceptance so as to cover most of the angular distribution). Here, we choose to normalize the experimental yields to the computed ${ }^{129} \mathrm{Te}$ cross section keeping the same constant for all other channels. One can see that the channels involving few-neutron transfers are quite well reproduced for both the pick-up and stripping.

The cross sections for the production of tellurium isotopes cannot be directly translated in cross sections for the heavy counterpart of the reaction, as nuclear evaporation acts differently for the light and heavy partner. Keeping this in mind, the obtained agreement with the GRAZING calculations for the Te isotopes allows us to show the predictions of the same code for the production of Au isotopes. These are displayed in the bottom panel of Fig. 9 here we show also the "production cross sections" in order to see the effect of evaporation, as estimated by the code.

We are perfectly aware that GRAZING is not a fully microscopic model in fact it incorporates the structure of the two reactants via some macroscopic models, surface phonons for the inelastic part and a collection of single-particle levels (close to the Fermi surface) for the treatment of nucleon exchange. This last is calculated by using semiclassical form factors (matrix elements) that are constructed from the set of single-particle levels. It will be desirable to have comparisons with "fully" microscopic theories that, incorporating some assumptions on the relative motion dynamics, are able to calculate cross sections for the different observables. Quite recently the time-dependent Hartree-Fock (TDHF) theory has been generalized [24-26] so to be able to calculate cross sections also for transfer channels. The application of this theory to our data could thus be important for the validation of our finding.

\section{CONCLUSIONS}

In this paper we illustrated our method to arrive at a determination of the mass of the heavy fragments in a reaction between quite heavy ions by employing a new dedicated set-up coupled to the PRISMA spectrometer which allowed to correlate the masses of the light and heavy transfer products via high-resolution kinematic coincidence. By comparing the experimental mass distributions of the heavy partner with simulations that incorporate the binary process and its subsequent de-excitation we could evidence the role of the evaporation in multinucleon transfer. The effect of the evaporation shows up as a bending of the mass centroids, that depends on the bombarding energy and on the projectile and target combination, in the mass-mass correlation matrix. The extracted total cross sections well agree with calculations performed with the GRAZING code down to several neutron transfers. The paper stresses that multinucleon transfer reactions provide a suitable mechanism to populate neutron-rich nuclei close to the lead region.

\section{ACKNOWLEDGMENTS}

The authors are grateful to the LNL Tandem-ALPI staff for the good quality beams and the target laboratory for the excellent targets. This work was partly supported by the 
European Union Seventh Framework Program FP7/2007-2013 under Grant Agreement No. 262010 - ENSAR. This work has been supported in part by the Croatian Science Foundation under project no. 7194 .
[1] C. H. Dasso, G. Pollarolo, and A. Winther, Phys. Rev. Lett. 73, 1907 (1994).

[2] V. Zagrebaev and W. Greiner, Phys. Rev. Lett. 101, 122701 (2008).

[3] T. Kurtukian-Nieto et al., Phys. Rev. C 89, 024616 (2014).

[4] M. Thoennessen, Rep. Prog. Phys. 76, 056301 (2013).

[5] R. A. Broglia and A. Winther, Heavy Ion Reactions (AddisonWesley, Redwood City, CA, 1991).

[6] L. Corradi, G. Pollarolo, and S. Szilner, J. Phys. G: Nucl. Part. Phys. 36, 113101 (2009).

[7] C. L. Jiang, K. E. Rehm, H. Esbensen, D. J. Blumenthal, B. Crowell, J. Gehring, B. Glagola, J. P. Schiffer, and A. H. Wuosmaa, Phys. Rev. C 57, 2393 (1998).

[8] S. Szilner, L. Corradi, G. Pollarolo, S. Beghini, B. R. Behera, E. Fioretto, A. Gadea, F. Haas, A. Latina, G. Montagnoli, F. Scarlassara, A. M. Stefanini, M. Trotta, A. M. Vinodkumar, and Y. Wu, Phys. Rev. C 71, 044610 (2005).

[9] L. Corradi, A. M. Stefanini, C. J. Lin, S. Beghini, G. Montagnoli, F. Scarlassara, G. Pollarolo, and A. Winther, Phys. Rev. C 59, 261 (1999).

[10] T. Mijatović, S. Szilner, L. Corradi, D. Montanari, G. Pollarolo, E. Fioretto, A. Gadea, A. Goasduff, D. J. Malenica, N. Mărginean, M. Milin, G. Montagnoli, F. Scarlassara, N. Soić, A. M. Stefanini, C. A. Ur, and J. J. Valiente-Dobón, Phys. Rev. C 94, 064616 (2016).

[11] Y. X. Watanabe, Y. H. Kim, S. C. Jeong, Y. Hirayama, N. Imai, H. Ishiyama, H. S. Jung, H. Miyatake, S. Choi, J. S. Song, E. Clement, G. de France, A. Navin, M. Rejmund, C. Schmitt, G. Pollarolo, L. Corradi, E. Fioretto, D. Montanari, M. Niikura, D. Suzuki, H. Nishibata, and J. Takatsu, Phys. Rev. Lett. 115, 172503 (2015).

[12] A. Vogt, B. Birkenbach, P. Reiter, L. Corradi, T. Mijatović, D. Montanari, S. Szilner, D. Bazzacco, M. Bowry, A. Bracco, B. Bruyneel, F. C. L. Crespi, G. de Angelis, P. Désesquelles, J. Eberth, E. Farnea, E. Fioretto, A. Gadea, K. Geibel, A. Gengelbach, A. Giaz, A. Gorgen, A. Gottardo, J. Grebosz,. Hess, P. R. John, J. Jolie, D. S. Judson, A. Jungclaus, W. Korten, S. Leoni, S. Lunardi, R. Menegazzo, D. Mengoni, C. Michelagnoli, G. Montagnoli, D. Napoli, L. Pellegri, G. Pollarolo, A. Pullia, B. Quintana, F. Radeck, F. Recchia, D. Rosso, E. Sahin, M. D. Salsac, F. Scarlassara, P.-A. Soderstrom, A. M. Stefanini, T. Steinbach, O. Stezowski, B. Szpak, Ch. Theisen, C. Ur, J. J. Valiente-Dobón, V. Vandone, and A. Wiens, Phys. Rev. C 92, 024619 (2015).

[13] S. Szilner, C. A. Ur, L. Corradi, N. Marginean, G. Pollarolo, A. M. Stefanini, S. Beghini, B. R. Behera, E. Fioretto, A. Gadea, B. Guiot, A. Latina, P. Mason, G. Montagnoli, F. Scarlassara, M. Trotta, G. de Angelis, F. Della Vedova, E. Farnea, F. Haas, S. Lenzi, S. Lunardi, R. Marginean, R. Menegazzo, D. R. Napoli, M. Nespolo, I. V. Pokrovsky, F. Recchia, M. Romoli, M.-D. Salsac, N. Soić, and J. J. Valiente-Dobón, Phys. Rev. C 76, 024604 (2007).
[14] P. R. John, V. Modamio, J. J. Valiente-Dobon, D. Mengoni, S. Lunardi, T. R. Rodriguez, D. Bazzacco, A. Gadea, C. Wheldon, T. Alexander, G. de Angelis, N. Ashwood, M. Barr, G. Benzoni, B. Birkenbach, P. G. Bizzeti, A. M. Bizzeti-Sona, S. Bottoni, M. Bowry, A. Bracco, F. Browne, M. Bunce, F. Camera, B. Cederwall, L. Corradi, F. C. L. Crespi, P. Desesquelles, J. Eberth, E. Farnea, E. Fioretto, A. Gorgen, A. Gottardo, J. Grebosz, L. Grente, H. Hess, A. Jungclaus, Tz. Kokalova, A. Korichi, W. Korten, A. Kusoglu, S. Lenzi, S. Leoni, J. Ljungvall, G. Maron, W. Meczynski, B. Melon, R. Menegazzo, C. Michelagnoli, T. Mijatovic, B. Million, P. Molini, G. Montagnoli, D. Montanari, D. R. Napoli, P. Nolan, Ch. Oziol, Zs. Podolyak, G. Pollarolo, A. Pullia, B. Quintana, F. Recchia, P. Reiter, O. J. Roberts, D. Rosso, E. Sahin, M.-D. Salsac, F. Scarlassara, M. Sferrazza, J. Simpson, P.-A. Soderstrom, A. M. Stefanini, O. Stezowski, S. Szilner, Ch. Theisen, C. A. Ur, and J. Walshe, Phys. Rev. C 90, 021301(R) (2014).

[15] P. R. John, J. J. Valiente-Dobon, D. Mengoni, V. Modamio, S. Lunardi, D. Bazzacco, A. Gadea, C. Wheldon, T. R. Rodriguez, T. Alexander, G. de Angelis, N. Ashwood, M. Barr, G. Benzoni, B. Birkenbach, P. G. Bizzeti, A. M. Bizzeti-Sona, S. Bottoni, M. Bowry, A. Bracco, F. Browne, M. Bunce, F. Camera, L. Corradi, F. C. L. Crespi, B. Melon, E. Farnea, E. Fioretto, A. Gottardo, L. Grente, H. Hess, Tz. Kokalova, W. Korten, A. Kusoglu, S. Lenzi, S. Leoni, J. Ljungvall, R. Menegazzo, C. Michelagnoli, T. Mijatovic, G. Montagnoli, D. Montanari, D. R. Napoli, Zs. Podolyak, G. Pollarolo, F. Recchia, P. Reiter, O. J. Roberts, E. Sahin, M.-D. Salsac, F. Scarlassara, M. Sferrazza, P.-A. Soderstrom, A. M. Stefanini, S. Szilner, C. A. Ur, A. Vogt, and J. Walshe, Phys. Rev. C 95, 064321 (2017).

[16] J. V. Kratz, M. Schädel, and H. W. Gäggeler, Phys. Rev. C 88, 054615 (2013).

[17] E. M. Kozulin, E. Vardaci, G. N. Knyazheva, A. A. Bogachev, S. N. Dmitriev, I. M. Itkis, M. G. Itkis, A. G. Knyazev, T. A. Loktev, K. V. Novikov, E. A. Razinkov, O. V. Rudakov, S. V. Smirnov, W. Trzaska, and V. I. Zagrebaev, Phys. Rev. C 86, 044611 (2012).

[18] E. M. Kozulin, G. N. Knyazheva, K. V. Novikov, I. M. Itkis, M. G. Itkis, S. N. Dmitriev, Yu. Ts. Oganessian, A. A. Bogachev, N. I. Kozulina, I. Harca, W. H. Trzaska, and T. K. Ghosh, Phys. Rev. C 94, 054613 (2016).

[19] E. Fioretto, F. Galtarossa, L. Corradi, H. M. Jia, F. Collini, T. Marchi, G. Colucci, T. Mijatović, G. Montagnoli, D. Montanari, F. Scarlassara, A. M. Stefanini, E. Strano, S. Szilner, G. Pasquali, and J. Grebosz, Nuclear Instruments and Methods in Physics Research A (2018), doi: 10.1016/j.nima.2018.05.011.

[20] A. Winther, Nucl. Phys. A 572, 191 (1994).

[21] A. Winther, Nucl. Phys. A 594, 203 (1995).

[22] Program GRAZING, http://personalpages.to.infn.it/rnanni/ grazing.

[23] G. Pollarolo and A. Winther, Phys. Rev. C 62, 054611 (2000).

[24] C. Simenel, Phys. Rev. Lett. 105, 192701 (2010).

[25] K. Sekizawa and K. Yabana, Phys. Rev. C 88, 014614 (2013).

[26] K. Sekizawa and K. Yabana, Phys. Rev. C 93, 054616 (2016). 